\title{
Research on BIM Technology in Construction Safety \& Emergency Management
}

\author{
Xiong Gao ${ }^{1, a}$, Yonghong Chen ${ }^{2, b}$ \\ ${ }^{1}$ Faculty of Environmental Engineering, Kunming Metallurgy College, Kunming, China, 650033 \\ ${ }^{2}$ Faculty of Architectural Engineering, Kunming Metallurgy College, Kunming, China, 650033 \\ axiong_8789@163.com, ${ }^{b}$ chenyh7600@163.com
}

Key words: BIM, Safety Management, Emergency Management, Lifecycle

Abstract: Building information modeling (BIM) is one of the most promising recent developments in the architecture, engineering, and construction (AEC) industry. With BIM technology, an accurate virtual model of a building is digitally constructed. This model, known as a building information model and BIM plays a vital role in lifecycle of an engineering project, such as designing, construction, operation and maintenance. This paper introduces the concept of BIM technology and summarizes research on BIM in construction safety and emergency management in china and abroad, proposed establishing a sharing platform of integration safety and emergency information data based on BIM technology in the build lifecycle.

\section{Introduction}

Definition of BIM. In the 1970s, BIM (Building Information Modeling)is first proposed by Chuck Eastman, Ph.D. , of Georgia Tech College of Architecture and Computing : "Building information modeling integrates all of the geometrics and capabilities, and piece behavior information into a single interrelated description of a building project over its lifecycle. It also includes process information dealing with construction schedules and fabrication processes." ${ }^{[1]}$

NBIMS(The US National Building Information Modeling Standards Committee)has the following definition :

"A building information model (BIM) is a digital representation of physical and functional characteristics of a facility. As such it serves as a shared knowledge resource for information about a facility forming a reliable basis for decisions during its life-cycle from inception onward.

A basic premise of BIM is collaboration by different stakeholders at different phases of the lifecycle of a facility to insert, extract, update or modify information in the BIM process to support and reflect the roles of that stakeholder. The BIM is a shared digital representation founded on open standards for interoperability." [2]

The Characteristics of BIM Technology. Visualization, Harmony, Simulation, Optimization, The outputs of the drawing, it can present the shape of the building in 3D and simulate the construction sequence of a project, which can reduce rework, save time and cost, optimize management of the building construction and improve economic effect for all participants.

Visualization, the space stereoscopic 3D models, more intuitive, more images. the visual results not only can be used for rendering the display and report generation, and, more importantly, project design, construction, operation in the process of communication, discussion, decision making is done in the visualization of state.

Harmony, this aspect is the key content of construction and design units. The application of BIM technology is to integrate various related drawings on a multidimensional model, be clear at a glance, the problem is easy to find, can reduce a lot of coordination work.

Simulation: In the design phase, BIM is able to design something need simulation to simulate experiment, such as: energy saving, emergency evacuation simulation, simulated sunlight and heat conduction simulation, etc. Using 4D (in time) of BIM model can simulate the whole construction progress. 
Optimization, using $3 \mathrm{~d}$ model, the unreasonable place changes ahead of time, to the place where the collision implementation of collision avoidance, in advance of the difficult sub divisional work adjustment in advance, reasonable arrangement of construction plan, in order to shorten the construction period and reduce the engineering cost.

The outputs of the drawing, BIM is not to the people see more daily architectural design institutes of building design drawings, and some processing components of the drawings. But by building a visual display, coordination, simulation, optimization, can help the owner about the structure, water and electricity line drawings.

\section{Literature Review}

\section{Researches of BIM-based Safety Management}

Safety management in design phase. Rowlinson \& Hadikusumo(2002) ${ }^{[3]}$ proposed a integration of virtually real construction model and produced a design-for-safety-process (DFSP) tool. This will help safe engineers to identify safety hazards inherited during the building construction phase and select accident precautions needed to prevent the occurrence of accidents. Taiebat $(2011)^{[4]}$ established a framework of the safety design knowledge was embedded to BIM system. Jia Qi et al. $(2011)^{[5]}$ and Sijie Zhang et al. (2011) ${ }^{[6]}$ demonstrated the collection of security design knowledge in the BIM by AEC3 XABIO software and Solibri Model Checker(SMC)software. Mohammad Kasirossafar et al. (2014) ${ }^{[7]}$ proposed the BIM of integrated design and collaboration can be used to strengthen the construction site safety and safety planning based on BIM applications. Hongling Guo et al. $(2015)^{[8]}$ based on the obstacles to execute DFCS, the model integrating BIM and safety rules was proposed to identify the unsafe factors automatically. Kamardeen(2010) ${ }^{[9]}$ proposed a schematic concept of model for PtD(Prevention through Design) is shown in Fig. 1. The first step in $\mathrm{PtD}$ using BIM is hazard profiling of BIM model elements, which is carried out by integrating the IFC file of these elements and the PtD expertise in the PtD knowledgebase. In the second stage, high hazard rated elements will be recognized and safe design suggestions will be provided for design revisions. After the design has been revised for safety, a second set of suggestions will be provided for on-site control.

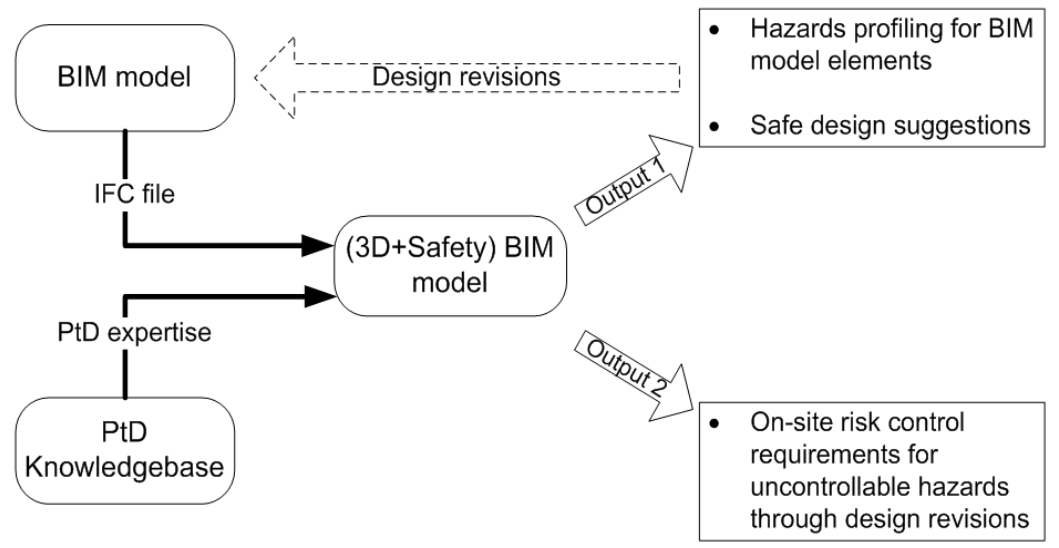

Fig. 1 : Framework for BIM-based PtD

Safety management in construction phase. Sijie Zhang et al. (2013) ${ }^{[10]}$ developed an automated safety rule checking to Building Information Models (BIM). Algorithms that automatically analyze a building model to detect safety hazards and what safety measures are needed for preventing fall-related accidents before construction starts. Through the integration of BIM, RFID location technology and network technology, H.L. Guo et al. (2014) ${ }^{[11]}$ proposed a model of a real time location and safety warning system for site workers , including BIM module, location module, data processing module, data transmission module and warning module . It is expected that this warning system can help to improve the efficiency of construction safety management and reduce the 
accident rate in the construction industry. Salman Azhar and Alex Behringer(2013) ${ }^{[12]}$ proposed the utilization of BIM technology can result in improved occupational safety by connecting the safety issues more closely to construction planning through a case study. This provides a more illustrative site layout, while providing methods for managing and visualizing up-to-date plans and site status information and visualization of the safety planning communication mode between the construction personnel.

\section{Researches of BIM-based Emergency Management}

Fire simulation and evacuation. Through micro-geographic information system (GIS) analyze 3D spatial data for fire-fighting simulations. Liang-Chien Chen(2014) ${ }^{[13]}$ established a 3D geometric network model (GNM)-based, building information model (BIM)-supported framework for fire-fighting simulation. Using the method, the best position for the deployment of the ladder trucks can be determined even before the arrival of firefighters at the scene. The proposed method can assist firefighters quickly locate their ladder trucks, thereby reducing response time after an incident. Xiaoyu Bai et al. (2013) ${ }^{[14]}$ set up a digital management system for fire emergency plan. These building modules information are obtained on BIM technology, which is superior to the traditional plans. In building model, it almost includes the entity information of building, such as fire equipment, the location and state of fire evacuation channels and fire compartments, etc. Then it can eliminate the cumbersome manual production process, simplify the compiling flow of current plans and improve the efficiency of the production plan combined with FDS, occupant evacuation software and the performance-based fire protection design, we can carry out the fire simulation drill and analysis to achieve the visualization and intellectualization plans and make the plan more reference and practical. Shih-Hsu Wang et al. (2015) ${ }^{[15]}$ designed a BIM-based model to support fire safety management of buildings. The model comprises four modules - evacuation assessment, escape route planning, safety education, and equipment maintenance. Yang Xuanfeng et al. (2013) ${ }^{[16]}$ classified the escape evacuation model is shown in Table 1, and the architectural BIM model was systematic analysised by escape simulation software. It got evacuation simulation dates that can help architects targeted adjustment and optimization to design .

\section{Table 1 Evacuation Model Classification ${ }^{[16]}$ )}

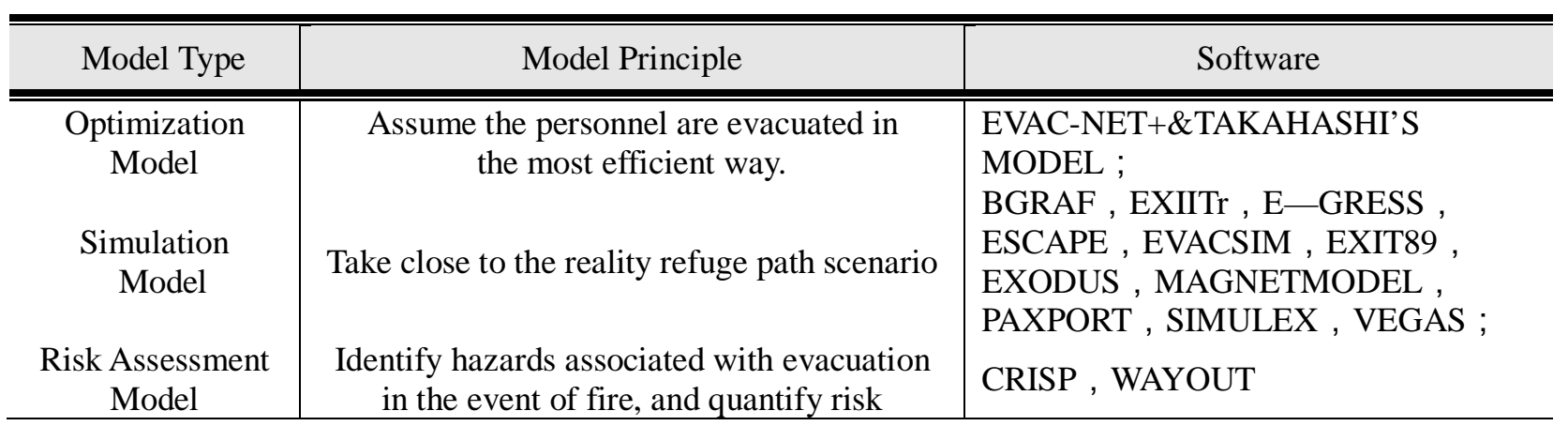

BIM and GIS. Tae Wook Kang(2015) ${ }^{[17]}$ proposed a BIM/GIS-based information Extract Transform, and Load (BG-ETL)architecture that separates geometrical information from that related to the relevant properties. The property required for each use-case perspective is extracted and transformed from information for BIM with FM to GIS using the ETL concept. Sam Amirebrahimi et al. (2016) ${ }^{[18]}$ presented an framework integrated BIM and GIS for utilization of detailed 3D building models for the Flood damage assessment (FDA) and 3D visualization of flood damage to building, according to its distinct behavior against flood. Yichuan Deng $(2016){ }^{[19]}$ used an instance-based method to generate the mapping rules between IFC and CityGML based on the inspection of entities representing same component in same model. Semantic City Model captures all the relevant information from BIM models and GIS models during the mapping process. 
Operation and Maintenance management. Chen Xinghai and Ding Lieyun (2014) ${ }^{[20]}$ established a system framework for the security operations management center of urban lifeline based on the IOT and BIM according the characters of the internet of things (IOT) and building information modeling (BIM), The system comprises five function modules-engineering information sharing platform, monitoring data management, 3Dsimulation, health diagnosis and safety assessment, emergency warning management. Zhao jian et al. (2015) ${ }^{[21]}$ established a 3D intelligent operation framework through the BAS(Building Automation System) is connected to BIM, it applied in the operation and construction of Nanjing Lukou international airport, The goal of smart airport is achieved by collecting, analyzing and managing data with BIM and integrating the monitoring and control of equipment, people management, maintenance management and emergency management etc . to a unified platform .

\section{Integrate safety\& emergency information data sharing platform of BIM-based}

Impacts of BIM on information source. The information transmission mode of BIM is minimized data loss in building lifecycle, traditional mode of information management is collected during each phase of the building lifecycle and lost at the handoff points to be partially recollected in many cases at additional cost. The solution is to use a standard framework to collect information as it is generated on a continuous basis. The way is integrating stovepipes and minimizing information loss from one phase of the life cycle to the next, thereby reducing overall costs for owners to operate and maintain the facility, as shown in Fig. 2.Information management mode based on BIM integrated of discrete information flow in building lifecycle and whole process, to avoid the information ambiguity and inconsistency, reducing the amount of information, information sharing and collaboration. This mode Can fully explores the information in all stages of the construction and realize highly efficient information transfer, exchange and sharing.

\section{Information Exchange Losses}

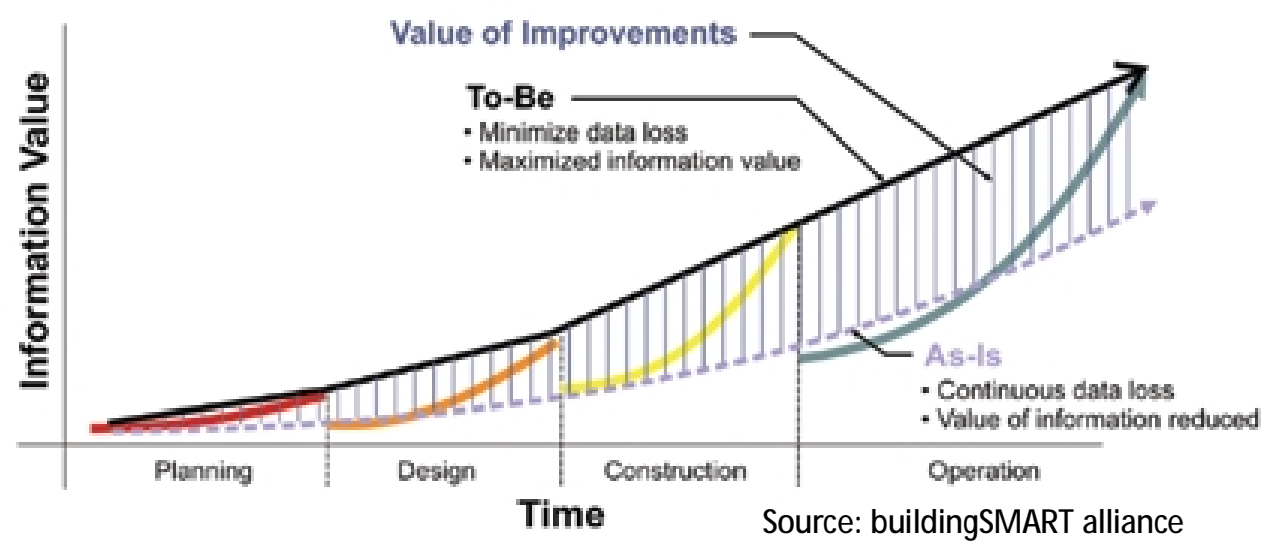

Fig. 2: BIM information seamless transmission

Integrate information data of safety\& emergency model. On the basis of summarizing the above research, the paper proposed to integrated model of BIM technology and safety and emergency management information in the building lifecycle, as shown in Fig. 3. These parameterization BIM information data of safety and emergency can be transformed and shared during each phase of the building lifecycle, which greatly reduces the distortion of the information transmission process.

The information and data of collecting from safety management platform can be transformed delivery to the emergency management platform, got emergency plan, which will be loaded by system, it can be demonstrated visual simulation in the BIM integration platform; and adjusted the emergency plan can also be fed back to the safety management platform, carry out virtual construction and simulation. 
In the simulation environment, the potential safety accidents are identified and the corresponding safety measures are formulated. In the building lifecycle, the BIM model integrated safety and emergency management information can be visualized on the construction personnel safety education and training, emergency evacuation simulation exercises, also can improved safety plan and emergency measures, from macroscopic to microscopic, abstract to concrete, static to dynamic, that will reduce the occurrence of accidents and improve capabilities of emergency response.

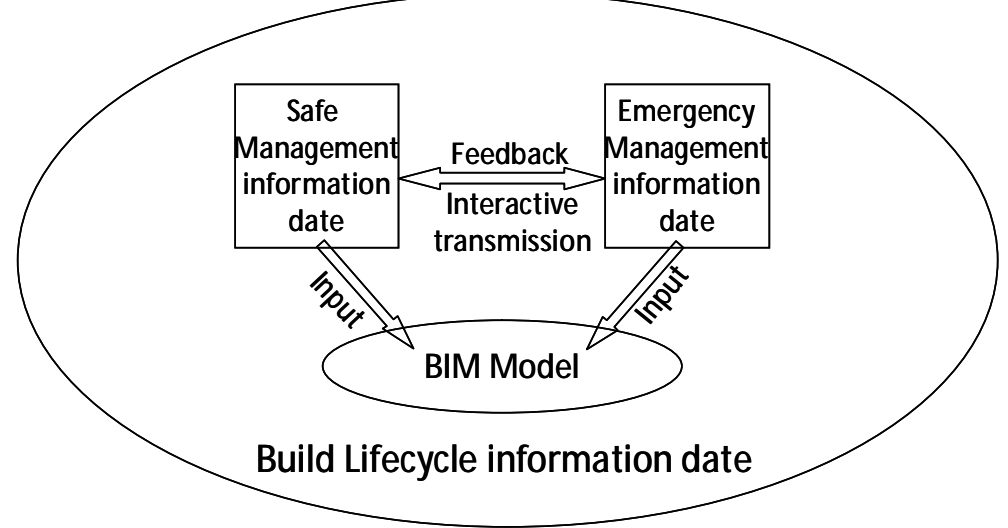

Fig. 3. Construction safe \& emergency information integration model based on BIM technology

\section{Conclusions}

Building information modeling (BIM) has recently attained widespread attention in the Architectural, Engineering and Construction (AEC) industry. Information integration based on BIM technology will play an important role in the building lifecycle safety and emergency management. In future cloud computing, big data and BIM will implement information sharing and integration, safety data automatically will be collected from a wide range of BIM users nationwide, it can find out regular of the accidents such as seasonality, periodicity and relativity, through analysis mass data in the construction process, and then we can make contingency plans and effectively reduce accident. Internet of things and BIM will deeper integrate, which is able to effectively identify and track dangerous construction process and locations, controlling methods and emergency moves can be applied in real time to ensure construction safety.

\section{References}

[1] Ballard, G., The Last Planner System of Production Control, 2000, Ph.D. Diss. , Faculty of Engineering, School of Civil Engineering, The University of Birmingham, UK .

[2] NIBS National BIM Standard Project Committee, National BIM Standard, 2006 , retrieved from http://llcic. vtt. fi/projectets/vbe-net/data/What is the NBIMS .pdf,5-12 .

[3]Hadikusumo, B.H.W., Rowlinson, S., 2002. Integration of virtually real construction model and design-for-safety-process data base. Automation in Construction.2002 . 11 (5), 501-509.

[4] Taiebat M . Tuning Up BIM for Safety Analysis Proposing Modeling Logics for Application of BIM in DfS[D] . Virginia Polytechnic Institute and State University . 2011 .

[5]Jia Qi1, R. R. A. Issa2, J. Hinze3 and S. Olbina . Integration Of Safety In Design Through The Use Of Building Information Modeling[J] . Computing in Civil Engineering . 2011 .

[6]Sijie Zhang , Jin-Kook Lee , Jochen Teizer , Charles Eastman . Integrating BIM and Safety: An 
Automated Rule-Based Checking System for Safety Planning and Simulation[C] . 2011 .

[7]Kasirossafar M , Shahbodaghlou F . Building Information Modeling or Construction Safety Planning[J] . American Society of Civil Engineers , 2014.

[8]Hongling Guo,Weisheng Zhang,Wenping Liu . Exploration on integration method of design for construction safety ( DFCS ) [J ] . Journal of Safety Science and Technology , 2015 ( 2 ) : 5-10 . [9]Imriyas Kamardeen . 8D BIM modelling tool for accident prevention through design[C]. //Proceedings of the 26th Annual ARCOM Conference, Leeds, September , Association of Researchers in Construction Management, Reading . 2010 , 1 : 281-289. [10] Sijie Zhang ., Jochen Teizer ., et al . Building Information Modeling (BIM) and Safety : Automatic Safety Checking of Construction Models and Schedules[J] Automation in Construction , $2013,29: 183-195$.

[11] Hongling Guo , Yantao Yu , Wenping Liu , Weisheng Zhang . Integrated Application of BIM and RFID in Construction Safety Management [J] . Journal of Engineering Management , 2014 , 28 ( 4 ): 087-092.

[12] Salman Azhar ,Alex Behringer .A BIM-based Approach for Communicating and Implementing a Construction Site Safety Plan . 49th ASC Annual International Conference Proceedings , 2013 [13] Chen, L.-C., C.-H. Wu, T.-S. Shen, and C.-C. Chou, The application of geometric network models and building information models in geospatial environments for fire-fighting simulations. Computers, Environment and Urban Systems, 2014. 45: 1-12.

[14] Bi, X.Y., J. Wang, and J.Y. Zhang, THE Exploration of BIM Technology Application in The Building Fire Emergency Plan, in Architecture, Building Materials and Engineering Management, Pts 1-4, H. Hou and L. Tian, Editors. 2013, Trans Tech Publications Ltd: Stafa-Zurich. p.2473-2477. [15] Wang, S.-H., W.-C. Wang, K.-C. Wang, and S.-Y. Shih, Applying building information modeling to support fire safety management. Automation in Construction, 2015. 59: 158-167.

[16] Yang Xuanfeng, Yan Wenkai, The Research of BIM-based Analysis about Evacuation Simulation. Journal of Information Technology in Civil Engineering and Architecture, 2013(3). [17] Kang, T.W. and C.H. Hong, A study on software architecture for effective BIM/GIS-based facility management data integration. Automation in Construction, 2015. 54: 25-38.

[18] Amirebrahimi, S., A. Rajabifard, P. Mendis, and T. Ngo, A framework for a microscale flood damage assessment and visualization for a building using BIM-GIS integration. International Journal of Digital Earth, 2016. 9(4): 363-386.

[19] Deng, Y.C., J.C.P. Cheng, and C. Anumba, Mapping between BIM and 3D GIS in different levels of detail using schema mediation and instance comparison. Automation in Construction, 2016. 67: 1-21.

[20] Chen Xinghai, Ding Lieyun, Research on operations management based on the internet of things and BIM of urban lifeline. Engineering Sciences,2014. 16(10): 89-93.

[21] Zhao Jian, Fang Jiankang, Song Yang, Chen Bangchang,Application of BIM Technology in the Operation of Nanjing Lukou International Airport. Journal of Information Technology in Civil Engineering and Architecture, 2015(2): 50-55. 